\title{
Chinese, Japanese and Korean-inspired Culinary Words in the English Language
}

\author{
JIEUN KIAER \\ University of Oxford, United Kingdom \\ NIAMH CALWAY \\ University of Oxford, United Kingdom \\ HYEJEONG AHN \\ Nanyang Technological University, Singapore \\ hjahn@ntu.edu.sg
}

\begin{abstract}
This paper discusses how Chinese and Japanese-inspired culinary terms have become part of the English language of food. It investigates the path of culinary terms into popular usage using Google Ngram, and Google Trends. It also takes an in-depth look at social media to discover the impact such technology has on the adoption of Asian culinary terms and further investigates this phenomenon by conducting a survey of 297 L1 and L2 English speakers, mainly from the United Kingdom. By selecting three specific kinds of culinary terms (tea words, dumpling words, and Yoshoku words), this paper showcases the creation of the forms and meanings of these culinary terms and documents their transnational journey to become part of English. The authors argue that the influence of social media, which has little linguistic authority, has made the process of the adoption of new words much more rapid, popularising their use and enabling diversity in their forms more than was ever previously possible.
\end{abstract}

Keywords: transnational; culinary terms; social media, hybridity, Asian languages

\section{INTRODUCTION}

With the opening up of East Asia to Western powers in the late $19^{\text {th }}$ century, the English lexicon experienced an influx of East Asian terms. Whilst this was on a smaller scale than the linguistic borrowing from English into Asian languages, there was a clear trend of words making their way into the English language, many of which can be traced to the present day. The majority of Asian-origin terms in the English language have originated from either Japanese or Chinese (Kiaer 2019). ${ }^{1}$ Japanese terms have been consistently entering the English language since contact began in the $16^{\text {th }}$ century via trading and political missions, with the majority of Japanese-origin terms entering a variety of Englishes being those pertaining to Japanese culture. Since the Meiji Restoration period of westernisation and modernisation in the $19^{\text {th }}$ century, contact with Japan has played a crucial role in introducing words of Asian origin into the English language. Words like tofu, tsunami, and haiku were introduced during this period (Loveday 1996). Most of these early words were nouns, with a large amount of them being culinary terms. Chinese terms entering the English language have faced a chequered history, with political turmoil throughout the late $19^{\text {th }}$ and $20^{\text {th }}$ centuries limiting the contact between the Chinese and English-speaking worlds. However, almost as a direct product of this situation, the earliest terms imported into English from Chinese were largely socio-politically distinctive words and culinary terms. The influx of terms from China is also diversified by the wealth of languages and dialects spoken in the region, which is especially noticeable in the comparison of English terms of either Chinese or Cantonese origin. This has resulted in a wide variety of pronunciations, spellings and translations occurring when these terms are accepted into the English lexicon. Whilst East Asian origin terms have entered the English language from 
countless fields and topics, those which show the greatest staying power, specifically those that have survived and thrived, are cultural words, especially those describing food (Bolton 2003).

This study aims to show how the use of Asian-origin and Asian-inspired culinary terms in the English language has come to take on diverse meanings and forms in recent years and demonstrates the role social media may have played in this transformation. These corpora include both historical and hybrid terms, with words such as tonkatsu, a Euro-Japanese term that emerged during the Meiji Restoration period of $19^{\text {th }}$ century Japan, and more modern hybrid terms such as sushi burrito or matcha latte. Focusing on three selected case studies, the main aims of this paper are to identify how and why certain culinary words originating in East Asian languages have been fully incorporated as elements of the English language, with a particular focus on the role of social media as a democratising and diversifying force. Data were collected from various sources such as Google Ngram and Google Trends as well as social media platforms such as Twitter. In addition to this, the results from 297 online survey responses are included. The results demonstrate that Asian culinary terms moving into the English language are varied in origin and form, with some being translated (such as dumpling), some being transliterated (such as sushi) and others being directly adopted hybrid terms (such as matcha latte). The results suggest that both the growing popularity of Asian food in online platforms and the linguistic freedom granted by online media are important factors in the development and flourishing of Asian-origin hybrid culinary terms in diverse ways, thus enriching the landscape of the English language.

\section{LEXICAL INNOVATIONS IN ENGLISH}

Lexical innovation as a natural localisation and nativisation process has been essential to research in studies of varieties of English. A proliferation of studies in new Englishes has focused on the hybrid nature of distinctive forms of localised Englishes that have emerged in consistent sociolinguistic and language contact situations. This includes early studies by Pride (1982), Platt et al. (1984), Kachru (1994) and more recent works such as Bolton's (2003) Chinese Englishes, Schneider's (2014) notion of "transnational attraction" in new Englishes and (2016) study of hybrid Englishes, as well as Kiaer and Bordilovskaya's (2017) three-stage process of English word borrowing in Japan and Korea. The foci of these studies are examining lexical variations and lexical borrowing processes. Kachru (1994), for example, classifies the processes of lexical borrowings into four main categories: namely, loan words, loan shifts, hybridisation and parallel lexical sets. Schneider (2014) argues that the Kachruvian model is limited in its ability to accommodate the vibrant and highly unpredictable nature of new Englishes developing from "emergent contexts" (2014, p. 24). He suggests the notion of "transnational attraction" (Schneider 2014, p. 28) to recognise the arbitrary nature of and the unbounded features of norms found in these Englishes. However, he has failed to provide a meaningful discussion on the notion of "transnational attraction" and how it can be applied to the study of new Englishes, and yet implies an unpredictable and almost untraceable mixing process would occur in new Englishes. Meanwhile, Kiaer (2019) showcases words with several translingual identities and provides a detailed case study on lexical integration, or mediation, occurring between East Asian languages and Englishes. The words that Kiaer (2019) examines reflect the notions of "transnational attraction". She suggests that these words "live across the borders of languages" (2019, p. 1) and they constantly travel and resettle in different languages, particularly through social media. In the pre-social media era, the local forms of English words were often produced by the mainstream media and linguistic authorities, and the new forms were largely limited to the influence of local language(s). However, the advent of social media has created highly individualized forms and meanings, whilst the ease of access offered by 
social media has boosted large-scale communication across different languages. This has resulted in the creation of new meanings and forms that are not only influenced by local languages but also by other languages. Therefore, the process of discovering how words in different languages are influencing the English language has become highly unpredictable and more complex than ever before.

While there is a large volume of studies investigating language contacts and their linguistic consequences within a wide range of contexts, few studies in new Englishes have focused on the transformation of culinary terms on the global stage. A small-scale study by Chimuanya and Awonuga in 2015 examines 34 culinary terms used in Nigeria and argues that the localised use of these terms demonstrates features of Nigerian English. Stanlaw (2004) examines the package labels of Japanese prepared foods and reports that the main functions of English loanwords for food in Japan are those of communicative strategies and graphical effects. In addition, the majority of influential culinary studies are centred on cultural and anthropological studies (e.g. Jurafsky 2014; Riley and Paugh 2018; Gerhardt et al. 2013), while little attention has been given to examining how social media has contributed to the birth of dynamic lexical creation processes and their outcomes in the global arena of culinary terms in English. The present study fills this gap by mapping the highly dynamic journeys of Asianinspired culinary terms into the English language and by examining the diverse semantics and forms demonstrated by these words and their usage on social media. The present study acknowledges that an in-depth examination of the transnational journey of the examined culinary terms requires hybrid and complicated lexical exchange processes which the concept of "lexical borrowing" in its traditional sense may be insufficient to explain. Hence, the present study does not use the term "borrowing" to depict the transnational journey by which new words are created.

\section{METHODOLOGY}

Three types of research instruments are employed in this study including online databases (Google Trends and Google Ngram), social media and an online questionnaire (Google Forms).

ONLINE DATABASES: Google Trends, Google Ngram

Firstly, the official definitions and usage statistics of the chosen words were established. The OED (Oxford English Dictionary) Online provided the definitions and dates of the words' entry into the English language, whilst Google Trends and Google Ngram online services had all the statistics relating to the use of chosen terms in certain regions and contexts. Google Trends also provided statistics for the frequency of online searches for a certain term. Rather than dividing by location, Trends can focus on one region (or "worldwide") and search for one or many terms, creating a graphic comparison of the frequency of searches for a number of terms. We have included regional breakdowns only for the cases in which we find it interesting to compare the locations where the terms are used with the localized origins of said terms (see Figures 4 and 6 for chai latte and matcha latte). This regional breakdown is not used in all cases as our aim for this paper is to trace the global popularity of terms in a brief and descriptive manner. A more detailed regional breakdown of globalised culinary terms on social media is something which we hope to look further into as part of future, more detailed studies. Google Ngram viewer provided the same kind of data as Google Trends, but based on the selected term's use in published books rather than online, giving information on the use of certain terms at earlier dates, before the advent of the internet and search engines. This enabled a comparison of the modern with the historical usage of East Asian culinary terms to be made. Whilst the corpus 
study was comprised of largely fruitful, reliable and readily available sources, there were some issues with this research method. The ambiguity in the meaning of certain terms worldwide meant that searching on Google Trends often entailed including words in different languages that were spelled the same but were not the words being searched for, hence potentially skewing the data. For example, results for the term mandu were unreliable as it is also the name of an ancient city in Madhya Pradesh, India, resulting in all searches on Google Trends becoming inflated by data irrelevant to the study. Furthermore, the limitations of Google Ngram are also acknowledged as it is only able to search books available online and so provides a rather limited sample rather than a full corpus search. Finally, these three resources are also limited by their time frames. Google Ngram is only searchable to roughly 1800 (based on the limited number of historical books that are available online compared to modern publications), whilst Google Trends only extends back as far as 2004, preventing a longer-term view of the usage of selected terms. Despite these limitations, it is believed that these sources provide useful information on the frequencies of words used online.

\section{SOCIAL MEDIA}

Twitter and Instagram were used because they are considered as the most ubiquitous and popular on the World Wide Web and have huge appeal to users with an instant global impact. ${ }^{2}$ These two platforms were also chosen due to the nature of their posts, with Twitter's lengthlimiting format encouraging brevity and spontaneity, and Instagram's visual element encouraging users to craft and present a certain image of themselves to the outside world, hence meaning that the two sites give similar yet nuanced sets of data with regard to the intention of the user. The chosen terms were sought out on these platforms to see how they are used in context. This involved searching for hashtags of the chosen words on said sites and taking screenshots of the posts in full, including details of usernames and time stamps. This provided good examples of the use of the chosen terms by real users as well as the evidence needed to back up the findings presented in the corpus study. Whilst a good source for research, this method is limited by the inability to see huge numbers of posts from a wide variety of dates at any one time, instead having to scroll down through posts ordered by popularity, recentness of publication, or a combination thereof.

\section{AN ONLINE QUESTIONNAIRE: Google Forms}

The final research method consisted of producing and distributing a questionnaire survey amongst L1 and L2 English speakers in various locations and backgrounds to gauge their opinions regarding certain terms. The survey received 297 responses and the majority of respondents (i.e. 161) were of British origin living in urban areas of the United Kingdom while others were from countries as diverse as Australia, Hong Kong, Singapore, France, Italy and Lithuania. All of those surveyed spoke English as either a first or second language, with roughly two thirds speaking multiple languages to at least a beginner level. As shown in Table 1 , the age distribution was relatively varied. 
TABLE 1. Demographics of participants

\begin{tabular}{cc}
\hline Age & $\mathrm{N}(\%)$ \\
\hline $11-20$ & $82(27.4)$ \\
$21-30$ & $94(31.5)$ \\
$31-40$ & $18(6.2)$ \\
$41-50$ & $35(11.6)$ \\
$51-60$ & $60(20.3)$ \\
$61-70$ & $6(2.1)$ \\
$71-80$ & $2(0.8)$ \\
Total & $297(100 \%)$ \\
\hline
\end{tabular}

The survey was conducted online via Google Forms during December 2018. Conducting an online survey using Google Forms was an effective method by which to gain a wide variety of responses from others on how they used East Asian loanwords, as well as enabling the researchers to gain an idea of demographical differences by including simple questions about the respondents' backgrounds. This was found to be a particularly useful research method as it prevented the authors from picking and choosing certain pieces of evidence that backed up original hypotheses. Instead, researchers were bound by the responses to adjust their hypotheses if required. The respondents were mostly speakers of British English, with the addition of some "native" Australian and US English speakers. Furthermore, a large proportion of the sample were affiliates of universities or educated professionals. Whilst survey respondents were asked to specify their general location (i.e. country), most did not disclose their specific regional location, such as town or city. This is not an obstruction to our study as our aim for this paper is to look at interregional comparisons (e.g. British vs American vs Australian vs Singaporean English) rather than intranational comparisons (e.g. London English vs Manchester English). This was a conscious decision on our part to maintain a tight scope in this preliminary paper on culinary words, and we hope to engage in a more fine-grained regional analysis in future studies.

The survey itself was comprised of six sections, the first five of which consisted of several questions within a given theme or format, and the last of which asked the respondents to give a little information about themselves (age, nationality, location, languages spoken etc.). The survey was advertised on social media and sent via email to a wide group of participants (who did the survey strictly by voluntary participation) based on the networks available to the authors and their connected institutions. It is taken on good faith that those responding to the survey were answering honestly and to the best of their ability, though of course this is not guaranteed. Whilst this factor harms the reliability of the questionnaire, we believe that the volume of responses and the questionnaire's nature of being relatively interesting but not concerning particularly divisive topics, allows us to say with relative confidence that the majority of answers are reliable. Particularly given that the responses are combined with our other sources of evidence, we believe this reliability to be sufficient for the given study.

In each individual case we have selected one or multiple data sources in our discussion of the transnational existence of the terms in question. The selection of specific sources in each case pertains to the nature of the term being discussed: those we consider to be internet born and bred will necessarily lean more on data collected from our social media study and Google Trends, whilst those with a longer history and for which we wish to investigate the origins we have relied more on tools such as Ngram viewer. We have tried to include survey results in our case studies as much as possible in order to corroborate our hypotheses with the opinions of the general public. 


\section{RESULTS}

Three types of culinary terms were chosen for investigation. These words were selected because they embody examples of the dynamic etymologies, transnational attractions and hybridity that are particular to this type of language transfer. These words are not considered to be representative of the influence of one language or of one particular culture, instead showcasing several cultural and linguistic connotations due to their transnational journey and representing extreme hybridity due to the influences of several languages. The first culinary term examined is tea alongside related words such as cha and matcha latte, forming a group of words exemplifying both transcultural and hybrid terms. The dynamic etymologies of these words are presented firstly, then followed by the survey results which shed light on their perceived cultural connotations. The second term under investigation are various words for culinary items under the umbrella term dumpling, including the related items baozi, jiaozi, gyoza, mandu, manti, momo and ravioli. The reasons for the popularity of some words over others are discussed, with an analysis of the term dumpling highlighting the interesting case of an established word in the English language (originally used to describe suet dumplings or Scottish clootie dumplings) gaining an almost entirely new connotation in modern English (that is, with reference to East Asian dumplings). The third group consists of Euro-Japanese yoshoku $\left(19^{\text {th }}\right.$ century Western-styled) food terms. These words are great examples of engrained hybrid words, with a particular focus on the word katsu as the most prevalent example in the English language.

\section{THE TRANSNATIONAL JOURNEY OF 'TEA’ AND ITS RELATED WORDS}

According to the OED, the word 'tea' entered the English language in 1655 and has a complex etymology. The term in English is a cognate for the same word in many European languages, including Spanish té, French thé, Italian tè, and Dutch thee, all of which are thought to have been born from the Malay term brought back from Bantam by the Dutch, or the alternative word in the Amoy (Xiamen) or Fujian dialects (茶 or te) as spoken in what was then Formosa (modern day Taiwan). ${ }^{3}$ Tea in the English language is accompanied by the parallel term cha, which originates from the same Chinese term, pronounced chá in Mandarin and Cantonese, and which most likely travelled from Macau via the Portuguese colonies, showing up in the now obsolete cognates of Spanish cha and Italian cià as well as the still-existing cognates of Arabic shay and Turkish chäy. The word tea, therefore, has a long transcultural history. It is unique in being relevant to so many cultures across the globe whilst sharing the same root name (with almost all global tea-drinking cultures using variants of the original Chinese 茶 pronounced either cha or te), making the examination of the cultures associated with the term tea in the English language very interesting. For our investigation into tea words, we shall be looking at the term tea itself as well as the more recently popularised chai latte and matcha latte as examples of the growth of tea-related words via online trends. This study was conducted using data from the results of our survey as well as data gathered from Google Trends in order to show both the popular understanding and the online presence of our chosen terms. We choose to focus on Google Trends data in this chapter as the two phrases, chai latte and matcha latte have both found popularity online, thus making a study of online usage the most relevant to our investigation. We then back up this online data with opinions from our own survey. We have also included captions from Instagram in the case of matcha latte to clearly exemplify the use of the word on social media in the manner to which we are referring.

Despite the transcultural journey of the word tea, as shown in Figure 1, the results of the survey indicate that 161 respondents associate the word tea with an English word describing a British beverage, followed by a Chinese drink (118) and then a beverage of Japanese origin 
(51). The smallest number of responses (15) indicated "Other" or "Indian" as the origin of the word tea (13 people in total).

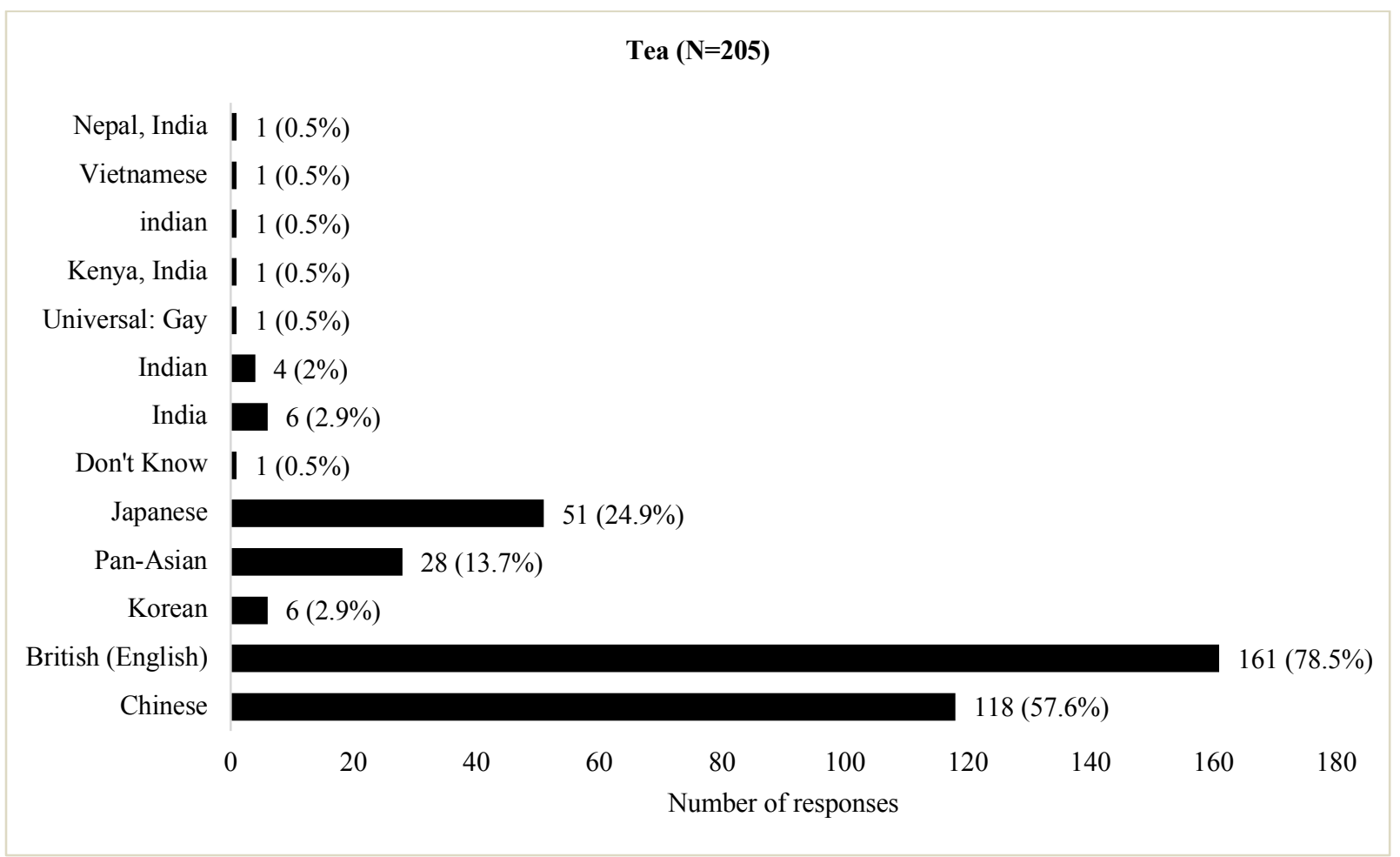

FIGURE 1. Survey results for the question 'Which language/culture(s) do you most associate with the word tea?'

Along with tea, the associated word cha was also investigated. Whilst cha was the first of the two to enter the English language in 1616, compared to 1655 for tea, it has since become a less common term, appearing only in British English and even then only in a slang context. ${ }^{4}$ This is probably due to the outdated nature of the term and its strong links to Britain's colonial past. This suggests that the terms tea and cha both exist in English, yet exhibit different connotations. A similar root is seen in the word chai, an adoption from the Hindi term which also originates from Chinese. This term is thought to have travelled from Chinese into Persian as chay, from which point it spread into Russian, Hindi, Arabic, and Turkic languages. Rather than entering the English language alongside the variant cha, the term chai did not formally enter the OED until the $20^{\text {th }}$ century, where it is listed as military slang and has since had additions proposing to list it as a word dating to 1957 that describes tea drinks associated with Arabic or Hindi speaking regions. In this context it is linked to the compound term masala chai (1977) denoting "spiced tea, typically brewed with a mixture of milk and water and sweetened". 5

Draft additions to the OED also include the term chai latte, listed as "a hot drink similar to masala chai, made with spiced tea or a commercially produced concentrate and steamed milk" dating to 1994. Consequently, the term chai (as distinct from cha) has developed different connotations for tea and is arguably no longer a mere slang term, with most modern English speakers associating it with a spiced, sweet and/or milky drink originating in the Indian subcontinent. When asked to which language/culture they think the word chai latte belongs, as indicated in Figure 2, 47.5\% of survey respondents selected "Indian", 27.5\% chose "English", $12.2 \%$ chose "Chinese" and $6.1 \%$ chose "Italian". This shows that modern English speakers largely associate chai or chai latte with a spiced variety of tea, rather than with a term referencing tea in general. 


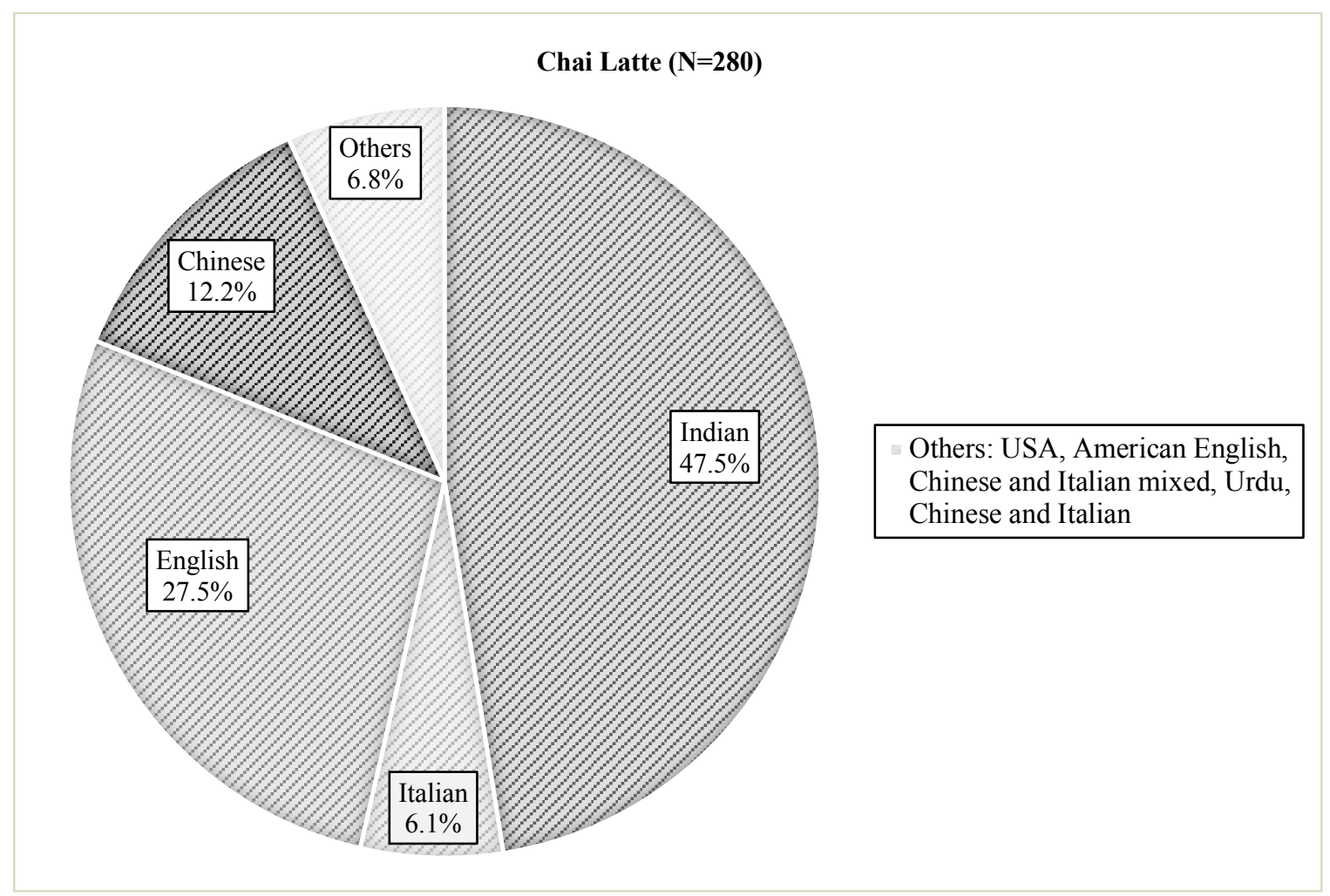

FIGURE 2. Survey results for the question 'To which language/culture do you think the word chai latte belongs?'

The Google Trends search for the term chai latte presented in Figures 3 and 4 shows how awareness and popular usage of the word increased online. This is evidence of the role that social media plays in shaping people's knowledge and opinions of certain foods and the words used to describe them.

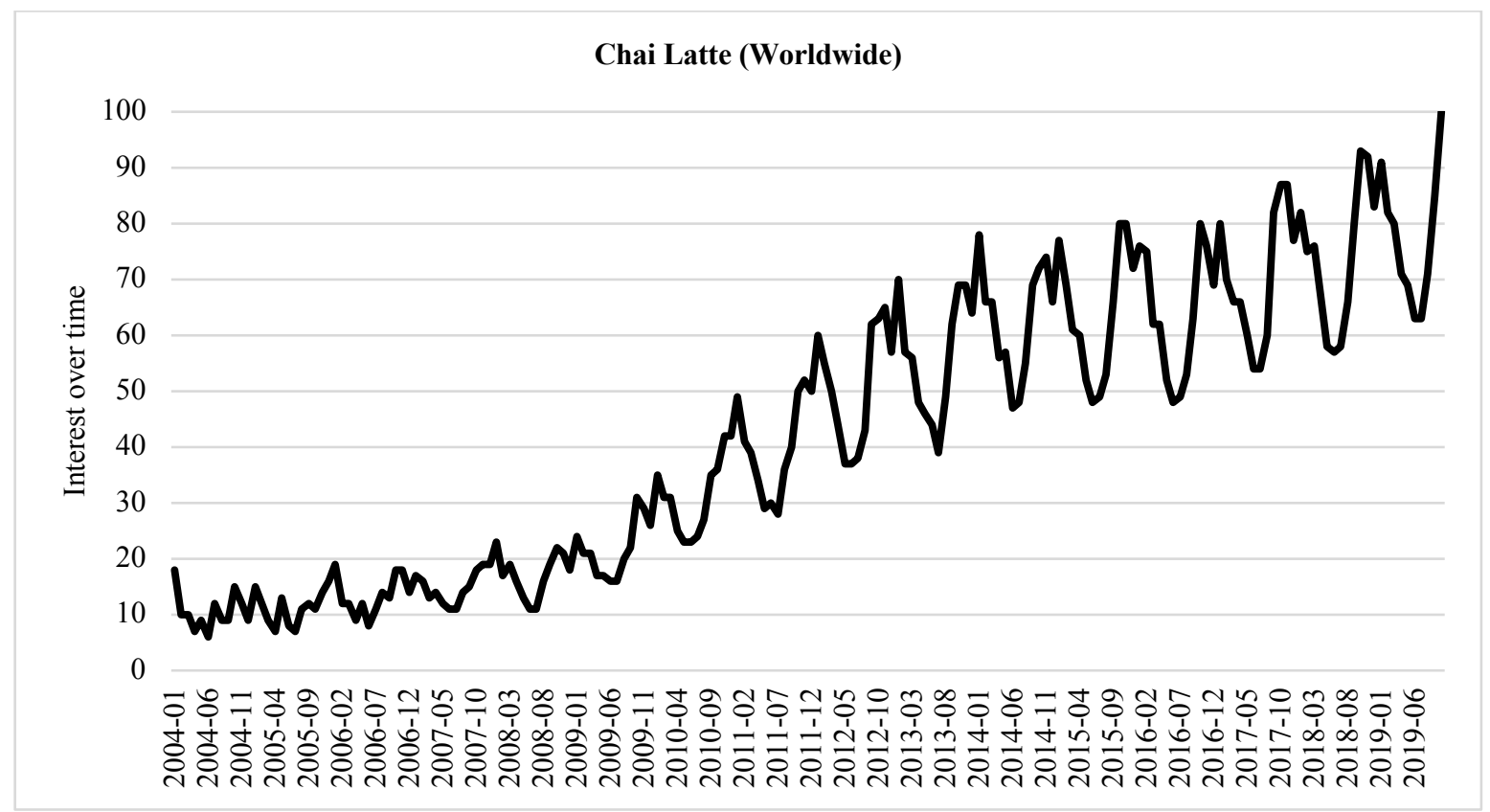

FIGURE 3. Google Trends results for chai latte, interest over time, worldwide, 2004-present 


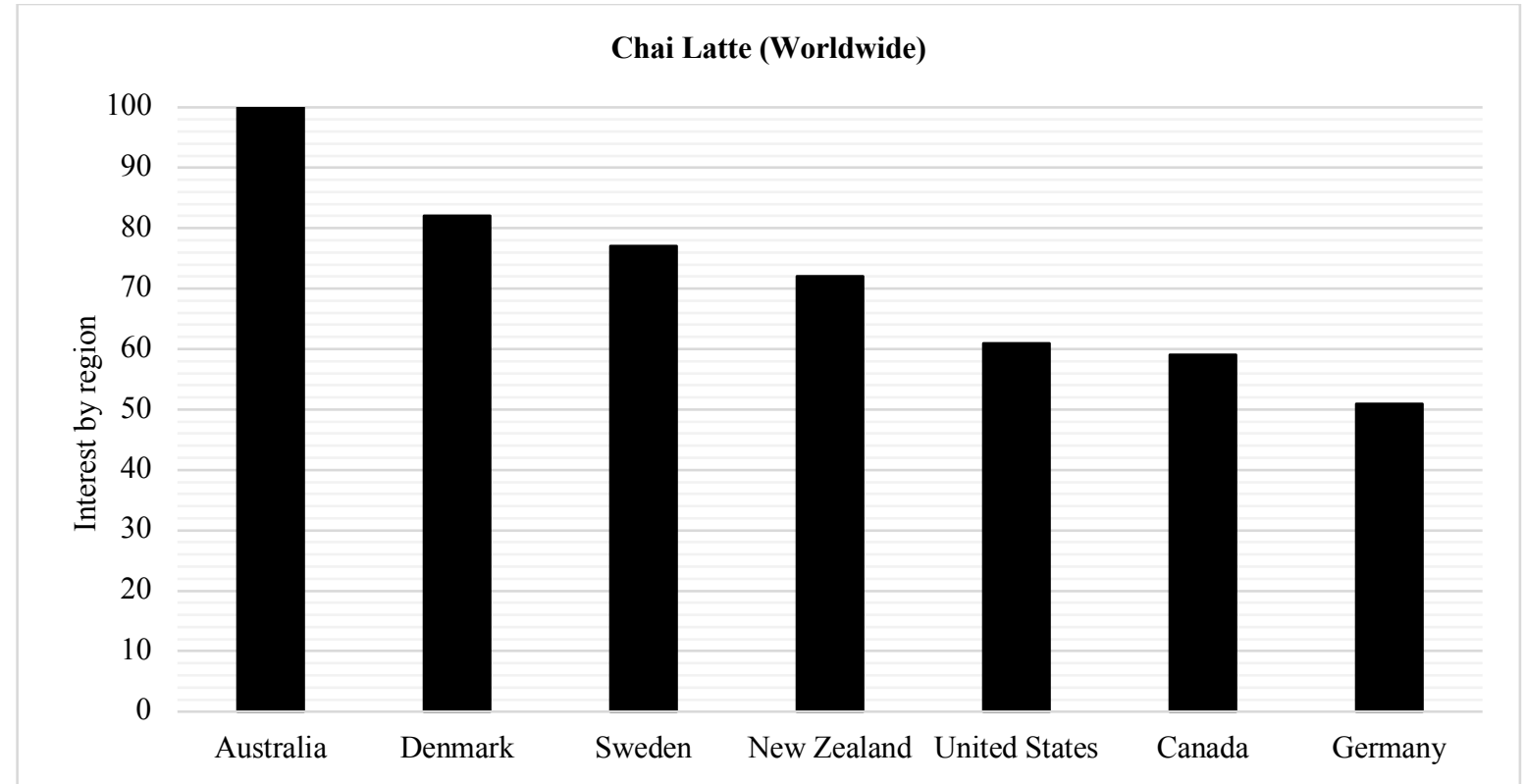

FIGURE 4. Google Trends results for chai latte, interest by region, worldwide, 2004-present

Figure 3 shows the increased use of the word chai latte over time since 2004. Numbers on the vertical axis represent search interest relative to the highest point on the chart for the given region and time. Values are calculated on a scale from 0 to 100 , where 100 is the location with the most popularity as a fraction of total searches in that location, while a value of 50 indicates a location which is half as popular. A value of 0 indicates a location where there was not enough data for this term. Thus, Figure 3 shows a steady increase in interest in the word chai latte over time. Figure 4 shows the results of interest by region. It shows which location the term was most popular in during the specified time frame. It is to be noted that a higher value means a higher proportion of all queries, not a higher absolute query count. So a tiny country where $80 \%$ of the queries are for "bananas", for example, will get twice the score of a giant country where only $40 \%$ of the queries are for "bananas". Figure 4 shows the top seven countries that most popularly use the word chai latte and indicates that chai latte is most popularly used in Australia, followed by Denmark, Sweden and New Zealand respectively. It also indicates that chai latte is popularly used in "Western" countries.

Another hybrid tea term investigated in depth is matcha latte. This word refers to a drink similar to a caffe latte, but instead of the coffee (or caffe) element, the drink is made with matcha green tea. When used together, the terms matcha and latte form a hybrid term describing an equally hybrid drink. The word matcha has fully entered the English lexicon, particularly in recent years as it has become a trendy foodstuff in the Western culinary scene. It is defined in the OED as "powdered green tea leaves which are added to hot water to make tea or used as a flavouring in desserts". ${ }^{6}$ Whilst the English term comes directly from Japanese, meaning a powdered green tea, its dictionary definition as a flavouring in desserts shows the importance of modern food trends in its development, revealing this to be the main form through which the word matcha has entered English. Despite its relatively long history in the English language (dating back to 1881), the term has only recently found a comfortable place in the English lexicon in its own right. This is evidenced by its various different spellings in English language publications (such as matcha, macha, maccha and mat-cha), as well as the habitual use of quotation marks and italicisation, indicating that the term has only recently been considered an English word worthy of special attention. This may well be attributed to the recent popularity of matcha as a flavouring in desserts and the increased awareness of Asian snacks in Western countries. 


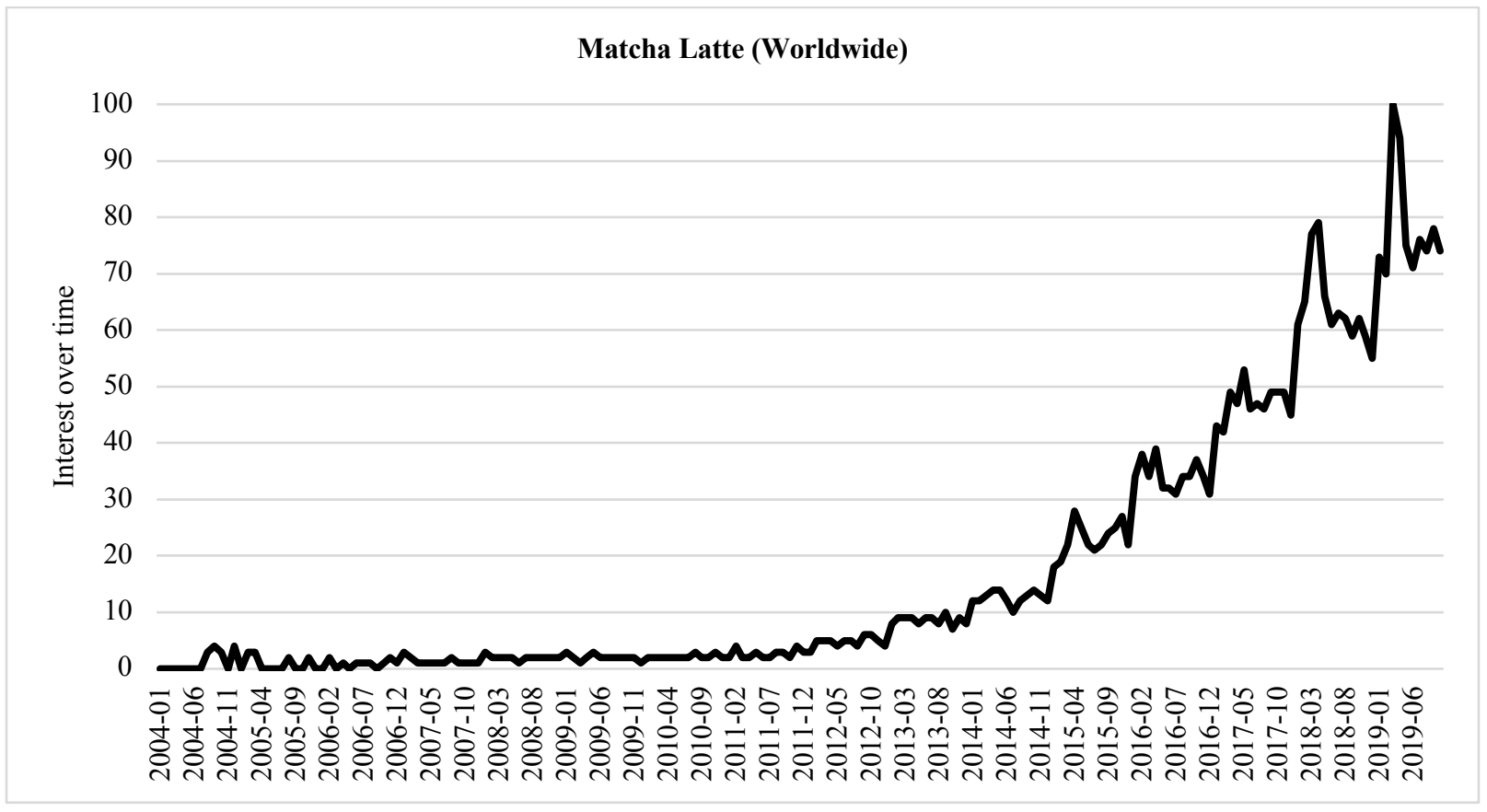

FIGURE 5. Google Trends results for matcha latte, interest over time, worldwide, 2004-present

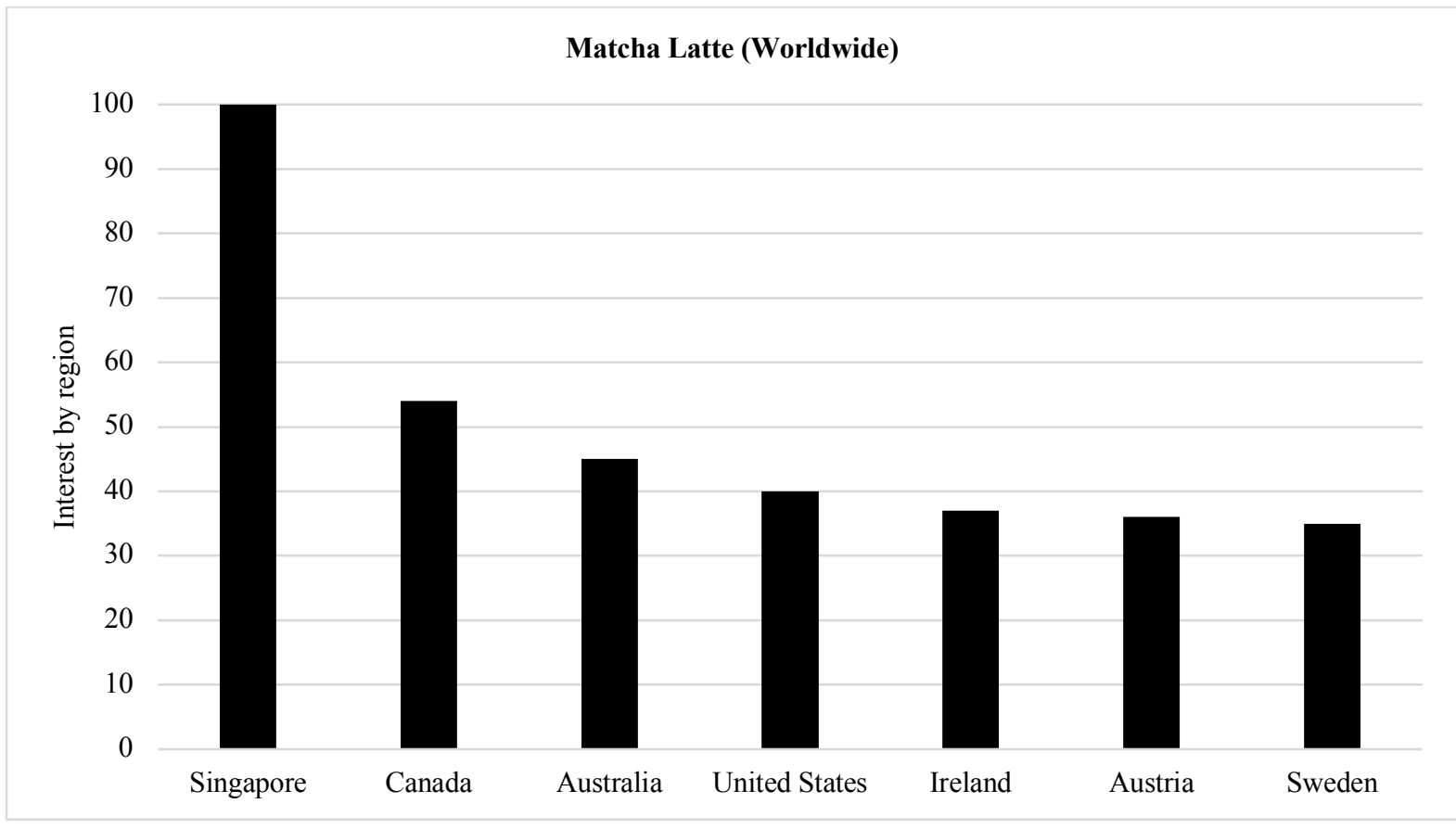

FIGURE 6. Google Trends results for matcha latte, interest by region, worldwide, 2004-present

Matcha latte is a particularly excellent term to examine as neither of the terms matcha nor latte are being used incorrectly. Whilst the term latte instantly makes one think of coffee, the word itself does not in fact have anything to do with coffee when considering its original Italian context, purely meaning 'milk'. This then leaves us with the question of which language we should consider the term matcha latte to belong to. One may initially consider it to be an English term due to its popularity and hybridisation in the West. However, the use of the term latte, stripped of its link to coffee, could allow us to consider it as being derived from the original Italian meaning, hence making matcha latte a direct hybrid of Japanese/Italian origin. 
There is yet another issue to consider: despite a matcha latte containing no coffee, the term still has clear origins in coffee culture and hence the term latte is adapted from its US English meaning, associating it with a kind of coffee drink, rather than the Italian meaning of 'milk'. US coffee culture has become increasingly popular in Asian countries and this may well be the origins of this hybrid drink. A search in Google Trends shows that the worldwide popularity of the term has been increasing exponentially since the late 2000s as shown in Figure 5, with Singapore being the most popular search region by far as shown in Figure 6 .

A search for the term matcha latte on social media shows most results to be related to "trendy" food items or café culture, indicating that this hybrid term is a product of internetbased food trends (See Figure 7). Most tweets and Instagram posts tagged with \#matchalatte are linked to Western-style café culture rather than being related to Japanese food or showing direct Japanese influence. This shows the term to be firmly rooted in the English language, with modern users associating it almost entirely with the English-speaking West and becoming desensitised to its Japanese or Italian origins.
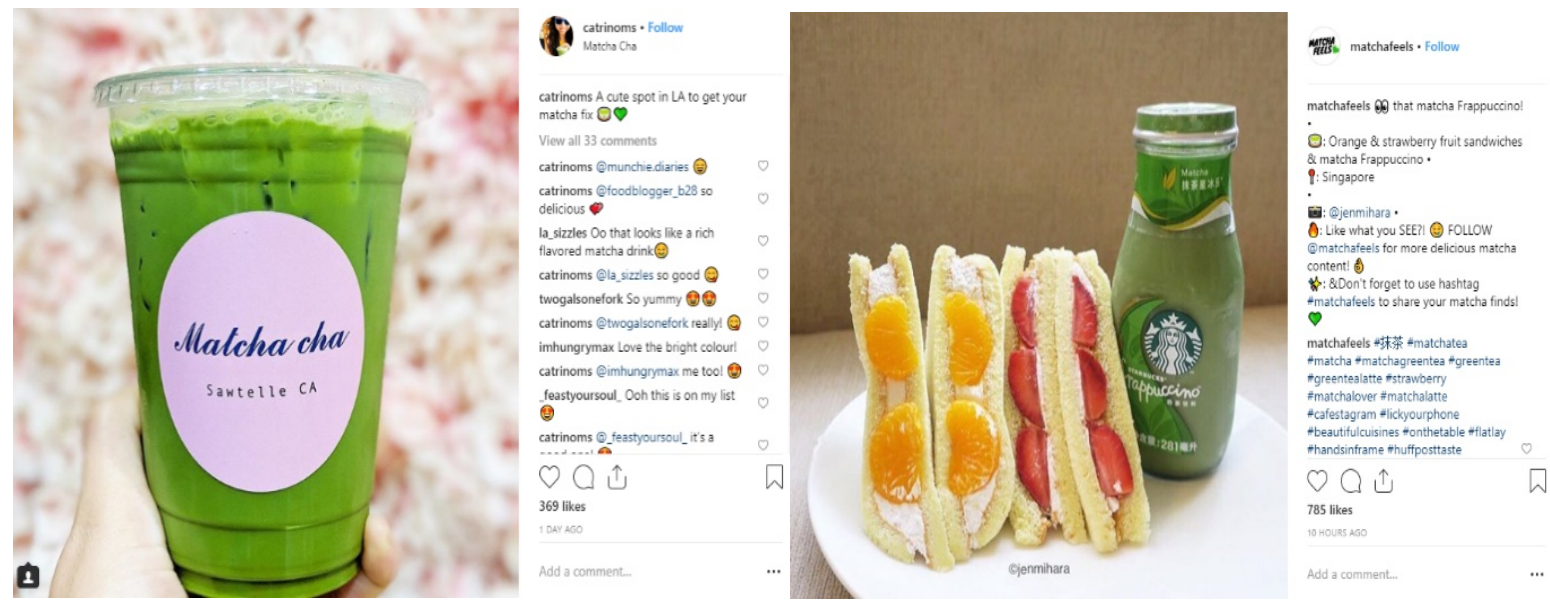

FIGURE 7. Some of the most popular Instagram posts including \#matchalatte

In the online survey, respondents were asked whether they considered the term matcha latte to be of Japanese, Italian, English or other origin. As shown in Figure 8 below, whilst the largest proportion of respondents suggested matcha latte to be Japanese $(57.2 \%)$, there were also a large number of participants who selected "English" $(21.4 \%)$ or "Others" $(8.7 \%)$ to specify US English as its origin. This suggests that even though the word "matcha latte" is often used in a Western context such as in a Western-styled café, the word itself is still strongly associated with Japanese origins. It is also interesting to note that a good proportion of people considered this hybrid term consisting of two foreign origin words to be English and some of them even linked it specifically to the US. This may indicate a heightened awareness amongst English speakers of hybrid food trends as an invention of the Western English-speaking world, thereby disassociating it from authentic international cuisines. 


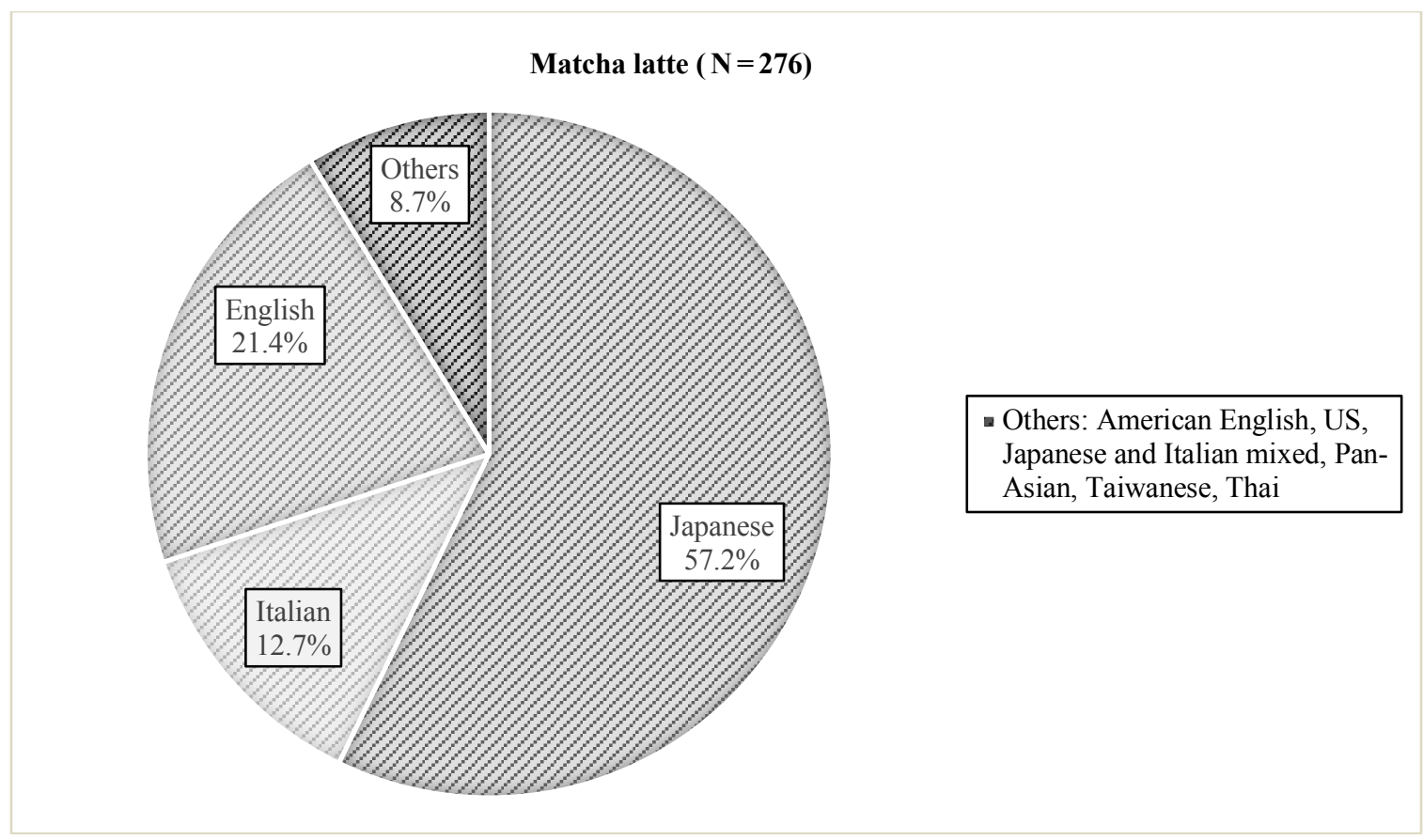

FIGURE 8. Survey results for the question 'To which language/culture do you think the word matcha latte belongs?'

In this section, several tea related terms were discussed. Firstly, the dynamic etymologies of the terms tea and cha were presented and the word tea was shown to be closely related to the concept of a British drink. Despite the late entry of tea compared to cha into the English language, the word tea is more popularly used in contemporary English. The interesting transcultural journey of the word chai was also noted, initially being adopted from a Hindi term that originated from Chinese. It only entered the OED in the $20^{\text {th }}$ century as a military slang term, and resurfaced in recent times with an additional meaning denoting spiced tea drinks, which has made it more popular. Then, the word chai latte was discussed in relation to the term masala chai. The term chai latte is used to denote meanings similar to masala chai, yet it is more popularly used in Western countries such as Australia and Denmark. According to the survey results, the word chai latte is associated with an Indian spiced variety of tea. The hybrid term matcha latte was also discussed in depth. Although matcha latte represents a trendy food item or Western-styled café culture, it is still closely associated with being Japanese and is most popularly used in Singapore.

\section{THE UMBRELLA TERM “DUMPLINGS”: JOURNEY FROM THE WEST TO BECOME AN ASIAN CULINARY TERM}

The second culinary term examined is dumpling and its related words. The word dumpling is an English language term that has been used to describe a huge variety of imported foods. With an OED definition of "a kind of pudding consisting of a mass of paste or dough, more or less globular in form, either plain and boiled, or enclosing fruit and boiled or baked (originally attributed to Norfolk)" or "a pasty mass like a dumpling" dating to around 1600, this term has evolved in the English language and become primarily associated with Asian foods. ${ }^{7}$ A search on Google Images produces results almost entirely comprised of Asian-style dumpling dishes, including Chinese baozi (steamed buns), jiaozi (thin-wrapper dumplings), guotie (fried dumplings or potstickers), Japanese gyoza (fried dumplings), Korean mandu (steamed or fried dumplings) and Nepalese or Tibetan momo. In fact, only four pictures are of dumplings that would not be considered "East Asian" in the first five pages of Google Images results, these being Austrian potato and pork dumplings (Hascheeknödel), an Anglo-American chicken stew 
with dumplings, Turkish manti and southern German bread dumplings. In our study of terms relating to dumplings, we begin with data from both Google Ngram and Google Trends. The former tool is used to help reveal the long and changing history of the meaning of the term dumpling in the English language, whilst the second tool aids us in reviewing the increasing use of transliterated regional terms, such as gyoza, in recent online discourse. We will then delve into a survey of dumpling-related social media posts alongside results of our questionnaire later in this study in order to trace the general trends of use and opinions amongst English speakers in the present day.

The term dumpling has been adapted to all kinds of Asian cuisines and is used with a certain ubiquity. It has come to describe any rounded dough-based item that either contains fillings or is empty, and that is cooked by almost any method (the most popular being steaming, boiling or frying). Whilst homogenously describing items that have been given different names in their original languages (such as Chinese baozi (steamed buns) and shui jiao (boiled dumplings)), the term dumpling has also evolved to cover all manner of hybridised and Westernised dishes. A Google search for dumpling recipes results in a wealth of hybrid dishes including "bacon cheeseburger dumplings", "Mom's special vegan dumplings", and "Asian vegetable dumplings". Dishes like this indicate two things. Initially, Western English speakers are comfortable with the term dumpling such that they see fit to adapt it to their own tastes. Secondly, using one word for a variety of distinct East Asian dishes has led to their homogenisation or "Pan-Asianisation". This can be seen in the wealth of online recipes described as "Asian style x" or "Oriental y", indicating how the adaptation of English-language terms to describe dishes has exacerbated a lack of awareness of the distinct nature of these cuisines.

Japanese gyoza, Chinese jiaozi and Korean mandu are three examples of the most representative dumpling-like items from each cuisine extant in the English lexicon. The terms jiaozi and gyoza entered the OED as late as 1965 and 1978 respectively (both with the exact same definition other than their country of origin), but a comparison in both online searches and published works demonstrates the overwhelming popularity enjoyed by the English term dumpling.

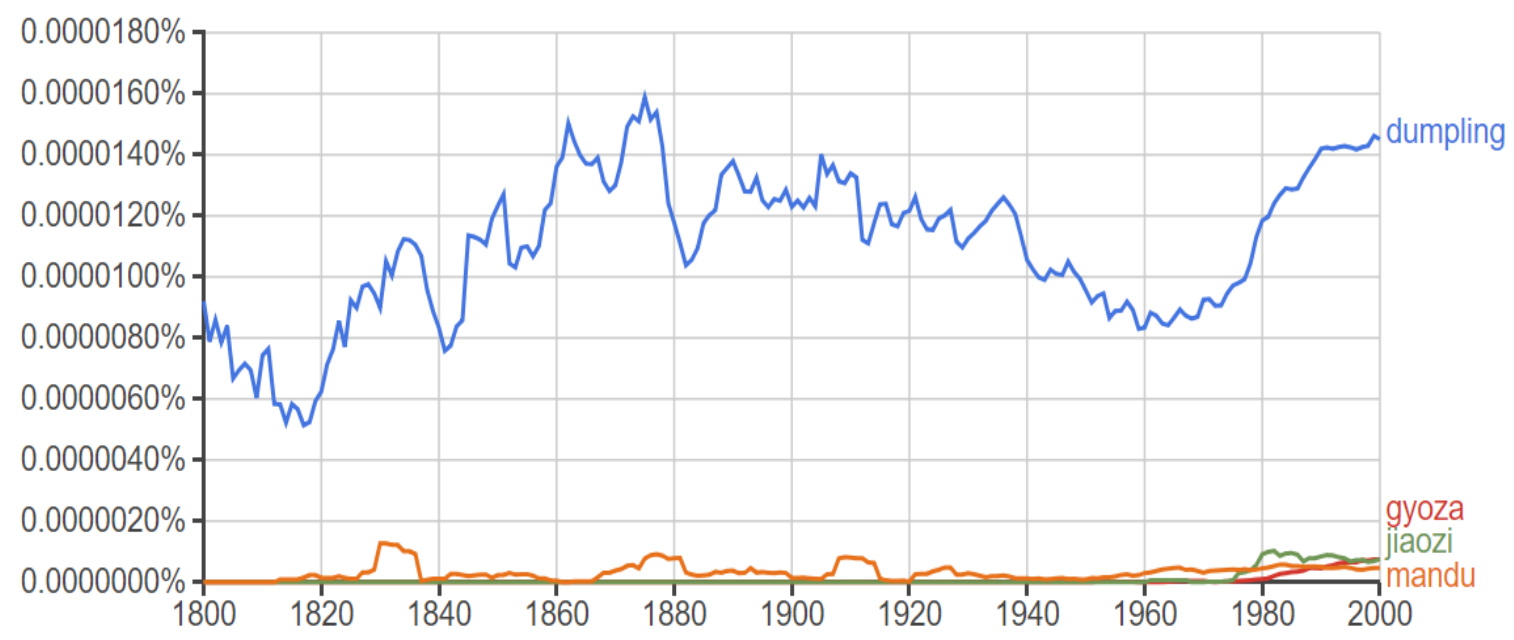

FIGURE 9. Google Ngram results for dumpling, gyoza, jiaozi and mandu, English corpus, 1800-2000 


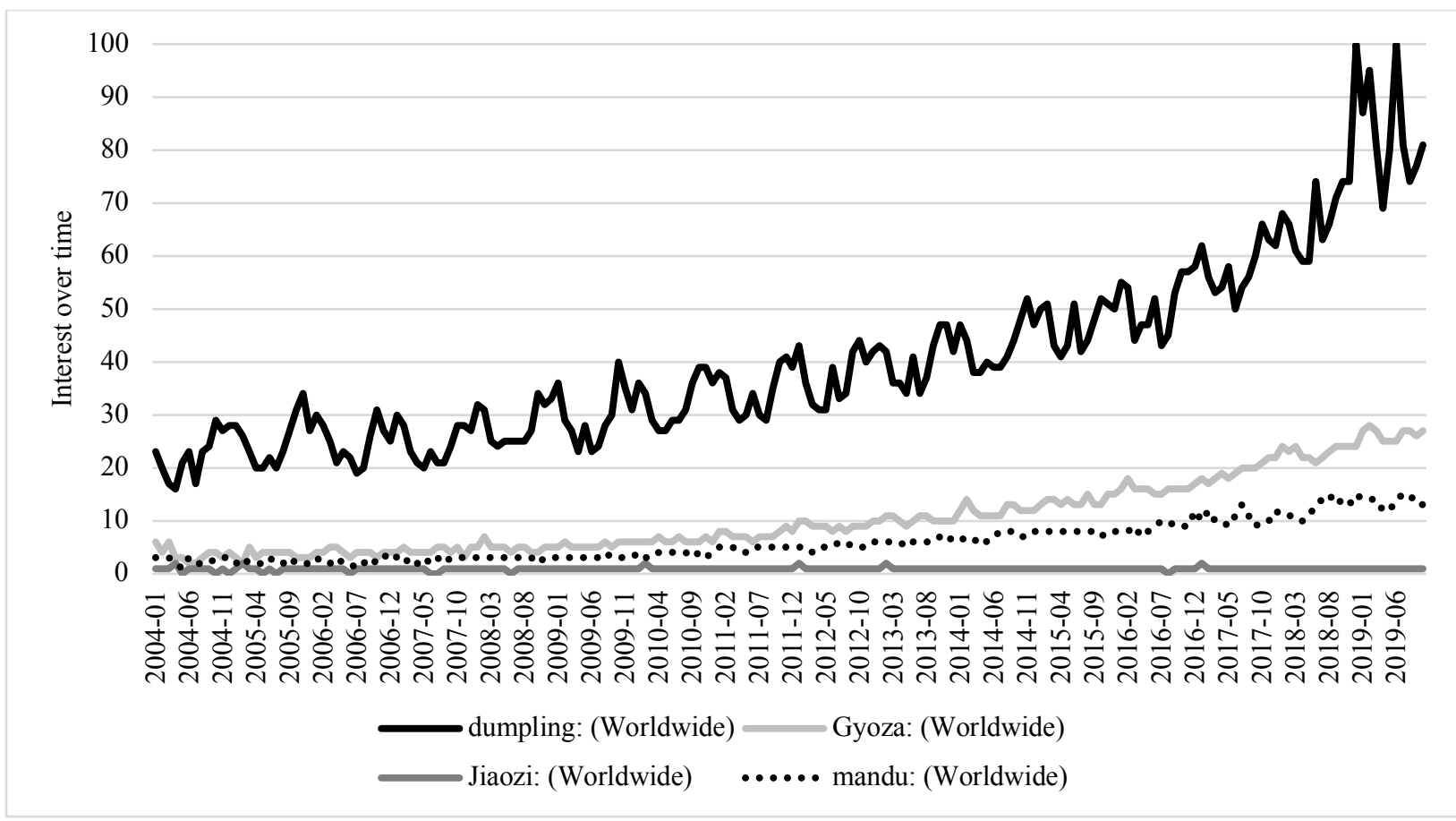

FIGURE 10. Google Trends results for dumpling, gyoza, jiaozi and mandu, interest over time, worldwide, 2004-present

In addition, Figure 9, the Google Ngram result, and Figure 10, the Google Trends result, indicate that the Japanese term gyoza and the Chinese term jiaozi, despite having the same characters (饺子) and OED definition, differ in popularity. The consistently higher use of the Japanese term, alongside its earlier official entry into the English lexicon, reflects a greater awareness of Japanese foods. This is most likely due to the longer history of connection between post-war Japan and the English-speaking West, as well as the perceived accessibility of Japan linguistically, culturally and politically compared to China. While the Japanese mostly recognise their gyoza dishes as originating in China, consumers in the English-speaking West seem more likely to be aware of the Japanese transliterated term rather than the original Chinese.

The dominance of the Japanese language in labelling Pan-Asian concepts (such as gyoza/jiaozi/mandu) can be seen in related posts on social media. Whilst many posts on both Twitter and Instagram feature \#gyoza without further explanation, the far less popular \#jiaozi and \#guotie (Chinese pan-fried dumplings) are used most often in combination with the word dumpling or other varieties of Asian dumpling-like foods by means of explanation. For examples, see the tweets below.

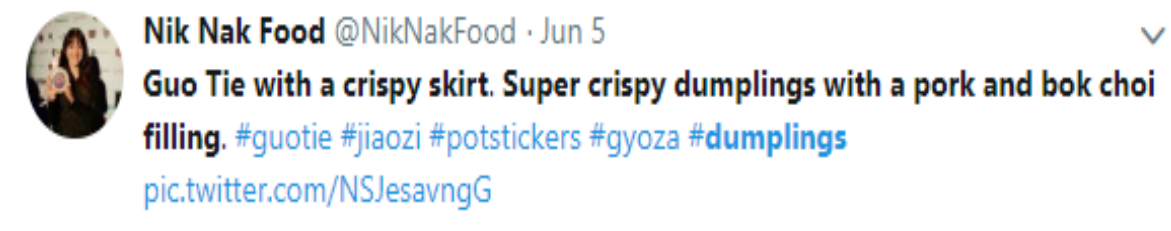

filling. \#guotie \#jiaozi \#potstickers \#gyoza \#dumplings pic.twitter.com/NSJesavngG

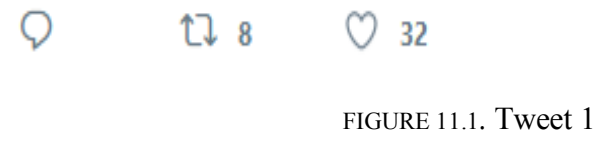




\author{
Food Cravings@FoodChefnicko.Sep 21 \\ Food Cravings - How To Make Chinese Dumplings (Jiaozi) Wonton Recipe \\ youtu.be/i9iUMKT7nAQ via @YouTube \#IAmACreator \#foodstagram \#jiaozi \\ \#clumplings \#chinesefood \#foofcravings \#chefnickostyle \#chinesedumplings \\ \#howto \#easyrecipes \#China \#zhangzhonjiang \#handynasty \#foodporn
}

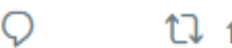

FIGURE 11.2. Tweet 2

The first tweet (Figure 11.1), shows a user describing a Chinese-style dumpling dish guotie (锅贴) using the qualifying terms jiaozi, potstickers, gyoza and dumplings, as the Chinese transliterated name alone is not enough to make the nature of the dish clear to the average English speaker. The second tweet (Figure 11.2), also demonstrates this, with the term jiaozi being put in brackets between the words dumpling and wonton, as these are clearly perceived to be terms more recognisable to English speakers.

A search for \#dumplings on Instagram also serves to prove the flexibility of the term and the breadth of cuisines it covers, as shown in Figure 12 below. Posts tagged \#dumplings include Chinese jiaozi, guotie, and sheng jian bao. Hong Kong char siu bao and har gow, Japanese gyoza, Korean mandu, Polish pierogi, Jamaican fried and steamed dumplings and Anglo-American sweet and savoury suet dumplings.

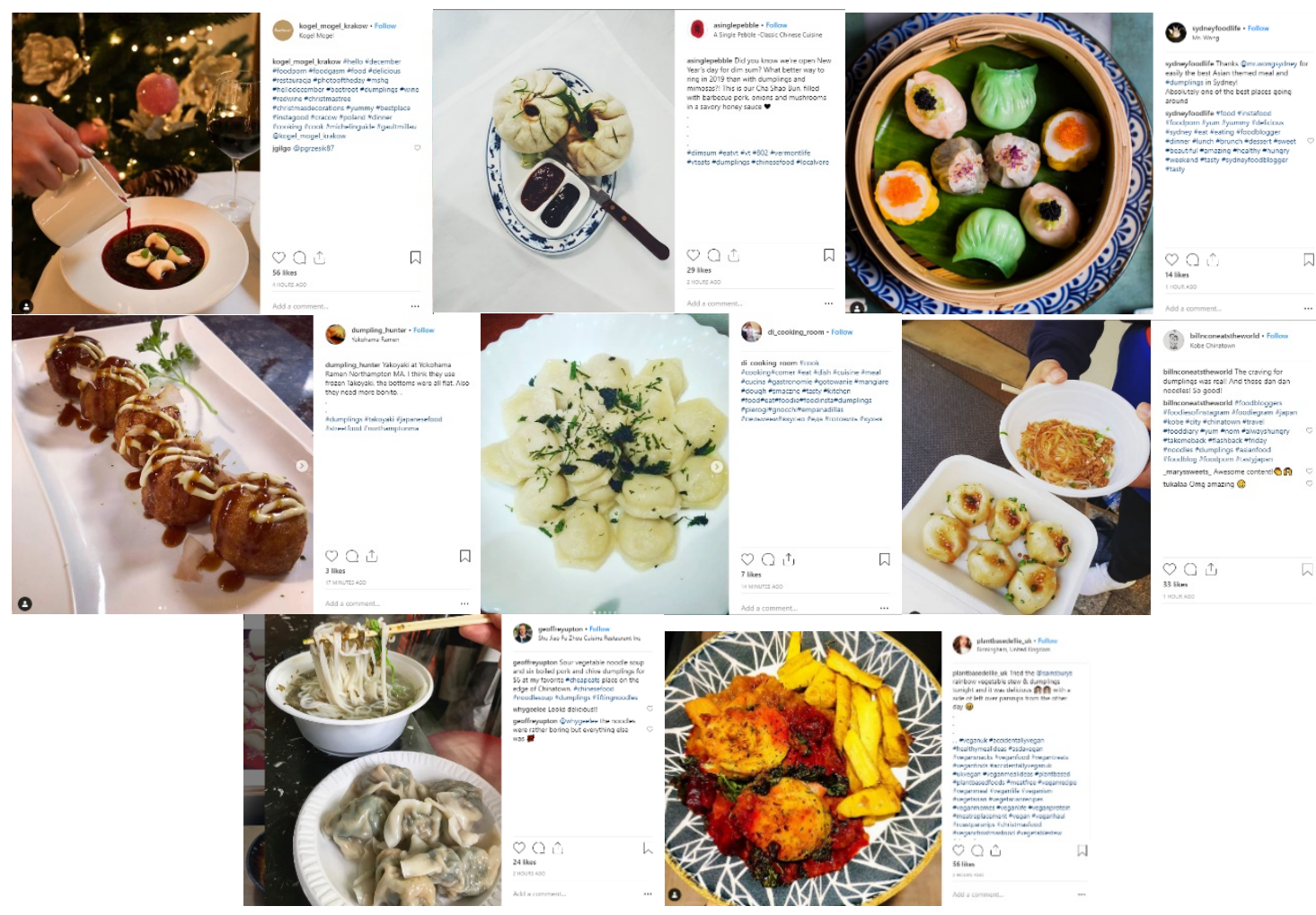

FIGURE 12. Instagram results for various kinds of \#dumplings

Whilst dumpling-like dishes of almost any culture are described as such in the English language, it is only Italian dumpling-like dishes that are rarely referred to as dumplings, with very few posts featuring the dumpling-like pastas such as ravioli, gnocchi, or tortellini showing up in social media and Google searches. Similarly, in British English, more so than in US English, Italian pastas have not been homogenised under the term noodle as have all the Asian 
varieties of what is effectively the same item. This could be considered a product of the West's longer history with and greater awareness of Italian culture, as well as the perceived greater authenticity of Italian food names. Where there has been a whole host of Asian foods homogenised under terms such as dumplings or noodles, the principal terms for Italian foods remain in the original language, such as pasta and pizza. This demonstrates the increased tendency to give generic labels to less familiar global cuisines by opting instead to use adapted terms from English that are more familiar.

Having surveyed the varieties of international dumplings in existence, the term has been wholeheartedly overtaken to represent East Asian varieties of the dish, almost totally losing its original meaning of Euro-American style steamed or baked puddings. A search of English language publications reveals that until the late $19^{\text {th }}$ century, the term dumpling was used almost exclusively in reference to Anglo-American style apple or suet dumplings, at which point it can also be seen in translated Asian literature and definitions of the word in Asianlanguage dictionaries. Even in the mid-20 ${ }^{\text {th }}$ century, when Asian dumplings seen in literature required no subsequent explanation as to what they referred to, cookbooks and recipes still seemed to only reference British, American and German-style dumpling dishes. This suggests that the exclusive adaptation of the term to Asian dishes is a far more recent phenomenon. As shown in Figure 13, the popularity of the word dumpling in English-language publications fluctuates, reaching one high in the mid-1800s, dipping during the mid- $20^{\text {th }}$ century and then picking up again towards the end of the century, embarking on a sharp incline towards a new high at the start of the $21^{\text {st }}$ century. The results suggest that these two peaks in the graph represent the two high points of popularity for dumpling in its two different meanings; the first referring to the traditional Anglo-American variety and the second representing the growing popularity of Asian dumplings since the late $20^{\text {th }}$ century.

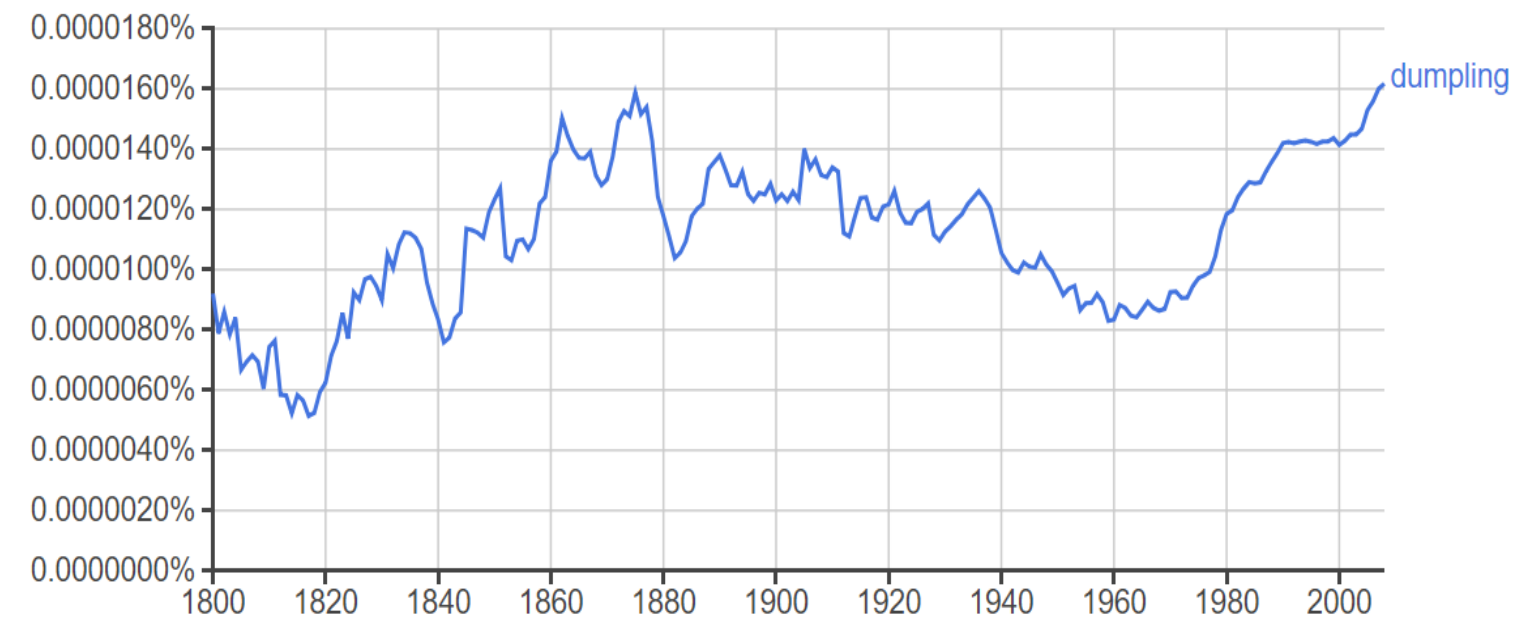

FIGURE 13. Google Ngram results for dumpling, English corpus, 1800-2008

Survey results also clearly attest to the most common understanding of dumpling amongst modern English speakers to be those of an Asian variety. When asked which language(s) or culture(s) they most associate the term dumpling with, as shown in Figure 14, the greatest response by far was "Chinese", being selected by $63.9 \%$ of those who responded. This demonstrates how the average modern English speaker most readily associates dumpling with being an Asian foodstuff. When later presented with seven images of different dumplings extracted from online searches as shown in Figure 15, 40.2\% of participants selected the Chinese baozi or steamed buns (option 1) and $29 \%$ selected the pan-fried jiaozi, gyoza or mandu (option 4). These results (69.2\% of responses overall), compared with only $21.3 \%$ 
selecting the Anglo-American style dumpling (option 5) demonstrates once again how Asianstyle dumpling dishes have taken primacy in the English-speaking mind, as shown in Figure 16. The recent nature of this change is also evidenced by the fact that most respondents who indicated the word dumpling to be British or selected the image of an Anglo-American stew with dumplings were those aged 40 and above, suggesting there to be a generational difference in the understanding of the term.

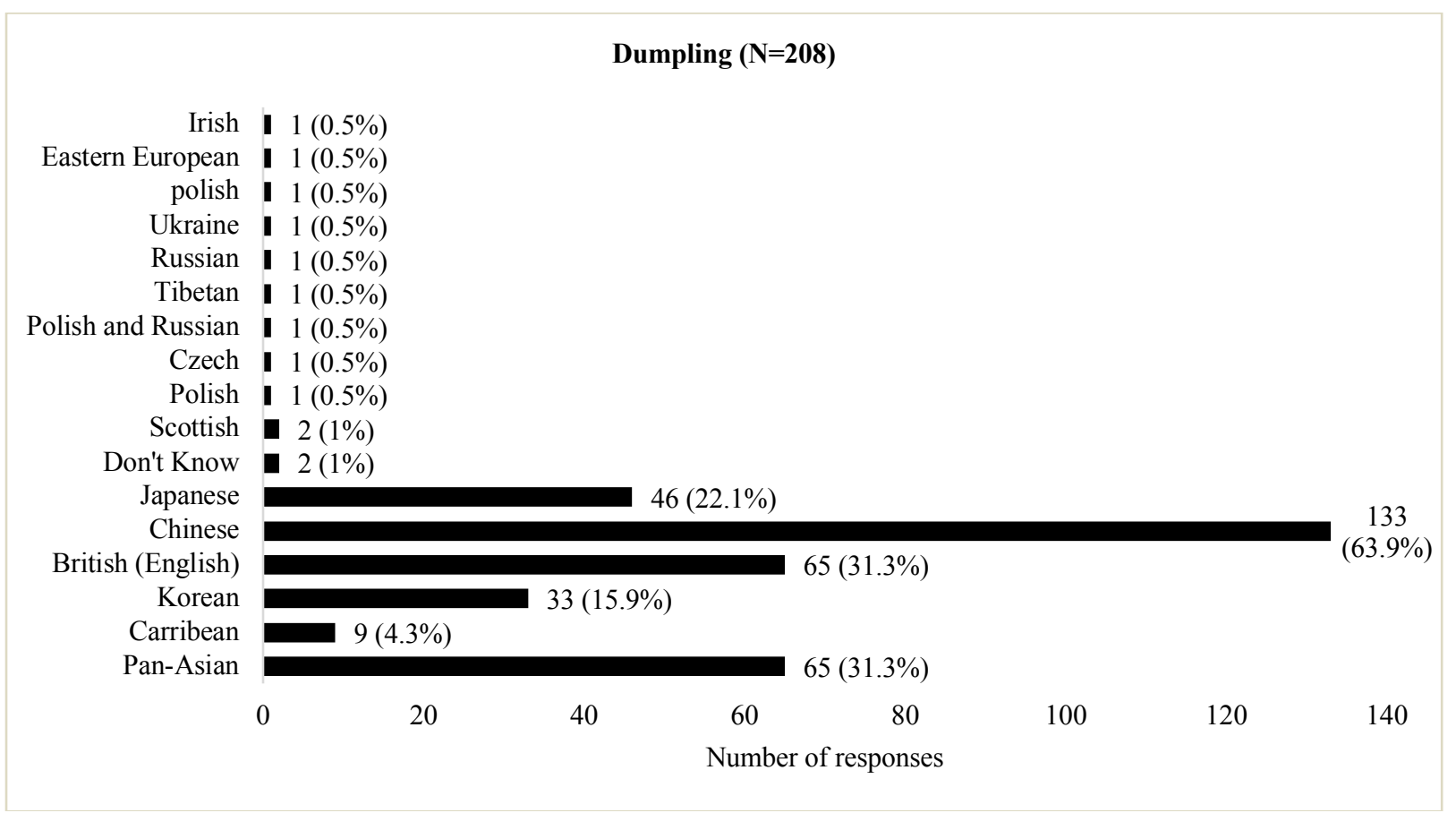

FIGURE 14. Survey results for the question 'To which language/culture(s) do you think the term dumpling belongs?'

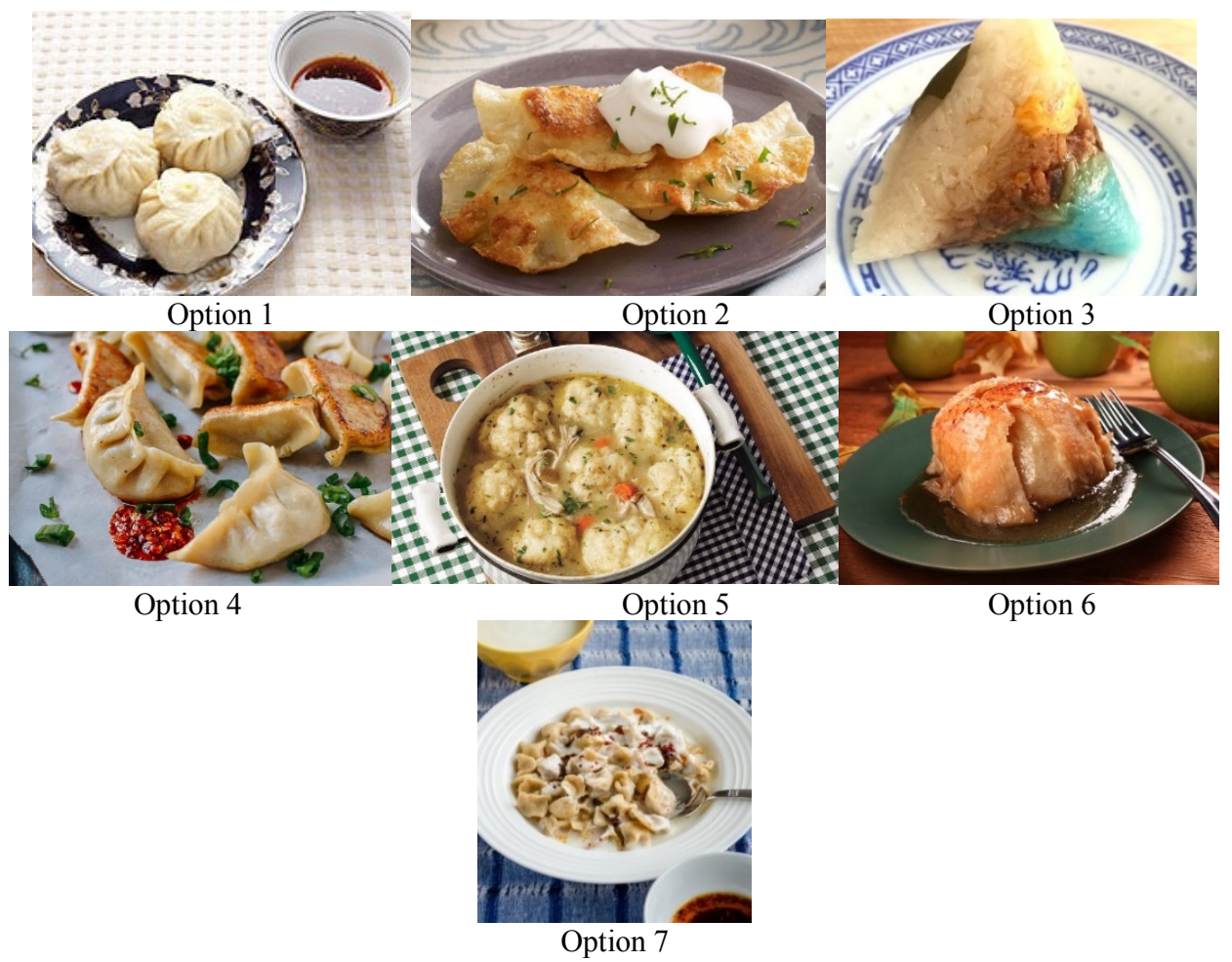

FIGURE 15. Survey options for the question 'Which image do you associate the most with the word dumpling?' 


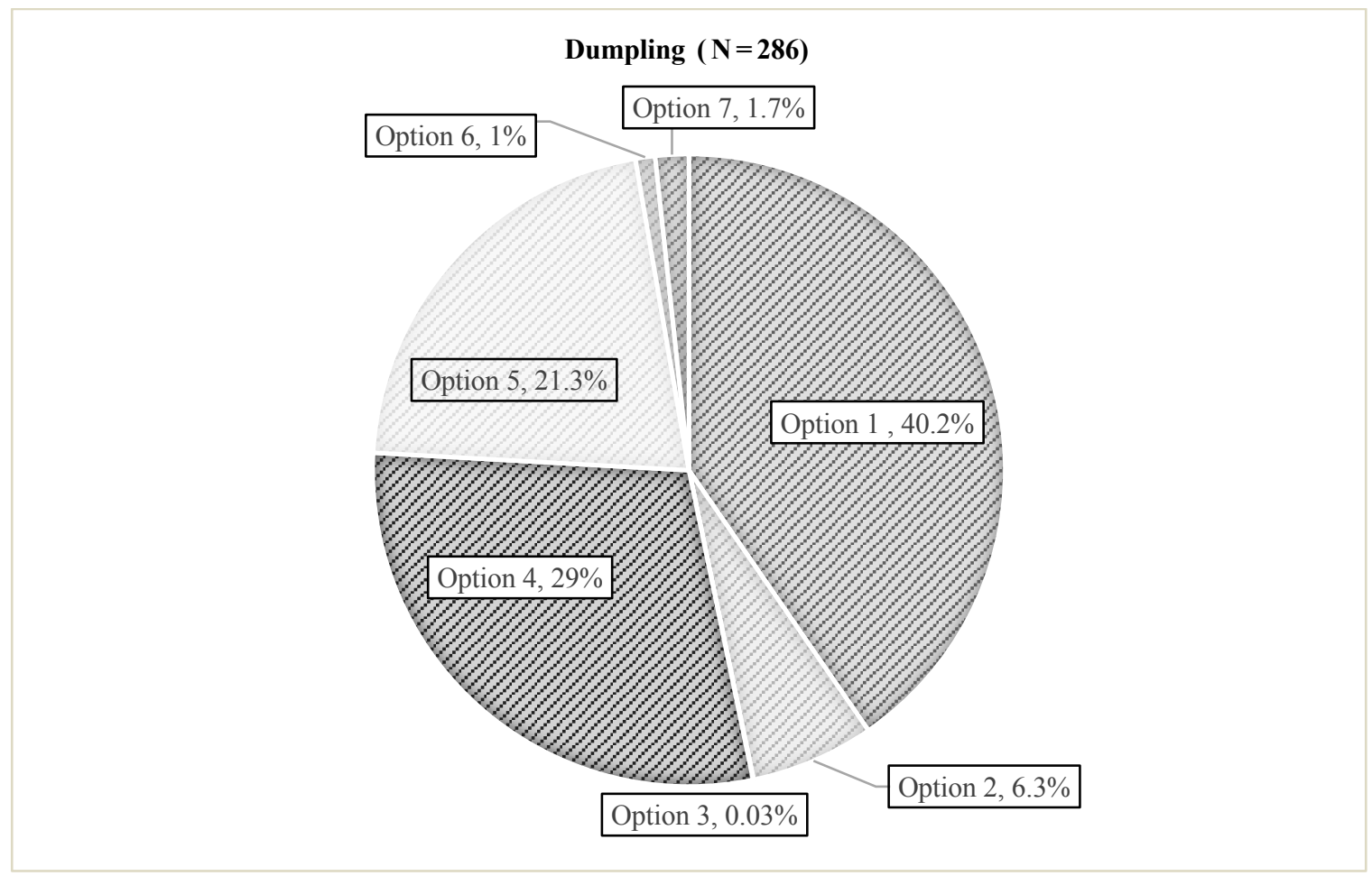

FIGURE 16. Survey results for the question 'Which image do you associate the most with the word dumpling?'

Furthermore, when given images of various dishes as can be seen in Figure 17 and asked to label them as if they were on a restaurant menu, one of the most common responses for both Chinese cha shao bao (or char siu bao, often translated as BBQ pork steamed buns) and a plate of fried dumplings that could be of Chinese, Japanese or Korean origin (that is, jiaozi, gyoza or mandu) on both counts was dumplings. This further illustrates the homogenisation of the term dumpling in referring to a variety of quite different Asian dishes.

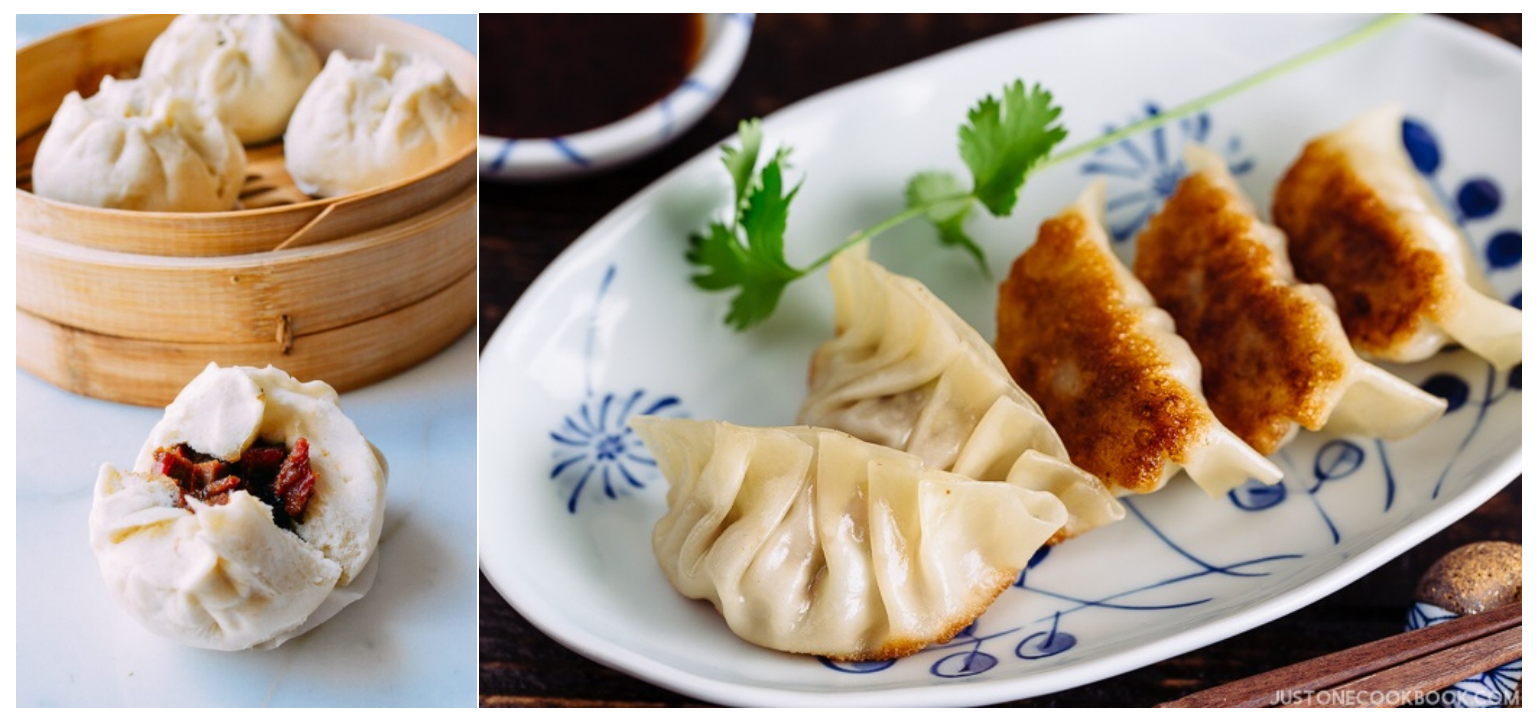

FIGURE 17. Two images provided for the survey question 'Imagine you are being served this dish in a restaurant, what would you refer to it as?' 
In this section, several terms related to the word dumpling are discussed in detail. Despite the word dumpling being attributed to originating in Norfolk in 1600, the contemporary use of a dumpling primarily refers to a type of Asian, pudding-like dish. The popular use of transliterated versions of dumpling, such as Japanese gyoza, Chinese jiaozi and Korean mandu, demonstrate the critical role that social media plays in promoting the use of transliterated versions of the term dumpling. While the Japanese term gyoza is more popularly used online without the aid of a definition, the Chinese word for dumpling, jiaozi, is used with qualification, accompanied by an explanation of what jiaozi are. Online searches for the word dumpling produces various dumpling-like dishes. Although the use of dumpling has consistently been popular since 1800 , the term dumpling before the $20^{\text {th }}$ century referred to a vastly different food than what is commonly accepted as a dumpling in the $21^{\text {st }}$ century. The survey results are also consistent with the online search which shows that a majority of respondents associate the word dumpling with Asian style dumpling dishes and the word dumpling is popularly used as an umbrella term to refer to a variety of different Asian dishes.

\section{YOSHOKU WORDS: JAPANESE DISH IN THE WEST OR WESTERN DISH IN JAPAN}

Yoshoku (洋食 meaning “Western food”) is a style of Western-influenced Japanese cuisine originating in the Meiji-era. These are Japanese versions of European dishes described with Western-origin names adapted and written in Katakana. ${ }^{8}$ As these food items became more popular, they are even treated like authentic Japanese dishes (和食 or washoku), served alongside rice and a soup and eaten with chopsticks. Katsu is a prime example of one such dish. Tonkatsu is a breaded and deep-fried pork cutlet, which is derived from Japanese ton (pork) and katsu, a shortened version of the term katsuretsu, a transliteration thought to come from a combination of European cognate terms for the word cutlet. This term is now a truly hybridised part of the Japanese lexicon and thus its adaption back into English makes it a "boomerang" word. ${ }^{9}$ In our study of the term katsu, our methodology relies on a study of Google Trends. Since the early history of the spread of Japanese terms such as katsu is already well documented, we have chosen to focus on online variations and uses of the term, particularly spelling variation, in order to demonstrate the flexibility of transliteration methods online.

As shown in Figure 18 below, a Google Trends search for various forms of the word katsu shows that, contrary to what is often the case, the transliterated version of the original term tonkatsu is more popular than the semi-transliterated term pork katsu, which could have been a more descriptive term for English speakers. However, the term chicken katsu is extremely popular in the English-speaking world, remaining on par with searches for tonkatsu, showing how the term and food item have been adapted to Western tastes. The popularity of the word tonkatsu (rather than pork katsu) attests to the increasing popularity of these items via social media and food trends. It is a more globalised culinary scene that causes transliterated terms like this to become part of the English language. 


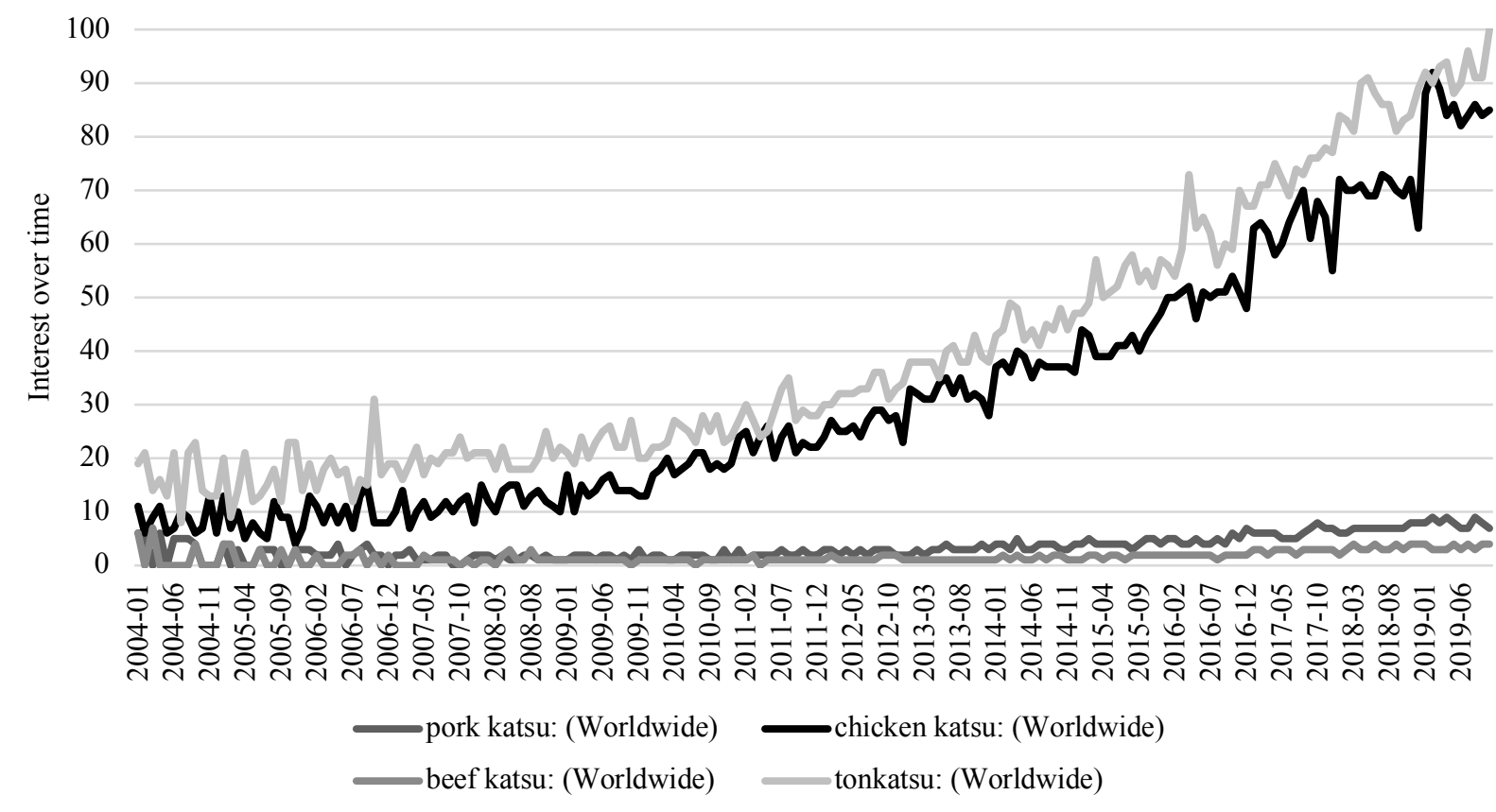

FIGURE 18. Google Trends results for pork katsu, chicken katsu, beef katsu, and tonkatsu, interest over time, worldwide, 2004present

\section{CONCLUSION}

This paper has observed how frequently culinary terms from Japan, China and Korea appear in the English language. Culinary words of foreign origin are constantly increasing and thriving in the English language largely due to globalisation, and these words often travel through social media. Along this journey, their forms and meanings take on transnational characteristics. The results of the survey and various big data methodologies have shown the variation in forms, the transcultural characteristics and the meanings of these terms. The advent of social media and its ubiquitous influence has made this process more diverse than ever before. That is to say, individual speakers choose words that lie beyond national, state and language borders. The less regulated channels of social media provide English speakers with the space to use language however they wish, regardless of their expertise, and be free of spelling, grammatical or syntactical barriers. In these spaces, words develop and change rapidly, enabling them to morph into huge numbers of variants and become popular at a breakneck speed. As Schneider (2014, p. 9) rightly points out the trend of "English being adopted by whatever means, in fragments, and unconstrained of norm concerns, driven by strongly utilitarian considerations", the present study casts light on the enormously dynamic adaptation of words into English in contemporary global society.

\section{ENDNOTES}

${ }^{1}$ The OED lists 1152 words from Central and Eastern Asian Languages, 533 of which are of Japanese origin, and 343 of which are of Sino-Tibetan origin.

${ }^{2}$ In the US, Instagram has 121.23 million monthly users and Twitter has 81.47 million monthly users, compared to 169.76 million monthly users on Facebook. (https://www.statista.com/statistics/248074/most-popular-ussocial-networking-apps-ranked-by-audience/). In the UK, Instagram has a $42 \%$ approval rating and Twitter has a $40 \%$ approval rating, compared to a $56 \%$ approval rating for Facebook. (https://yougov.co.uk/ratings/technology/popularity/social-networks/all).

${ }^{3}$ http://www.oed.com/view/Entry/198340?rskey=MrSwCs\&result=1\&isAdvanced=false\#eid 
${ }^{4} \mathrm{http} / / / \mathrm{www}$. oed.com/view/Entry/30117?rskey=xKKp2w\&result=1\&isAdvanced=false\#eid

${ }^{5}$ http://www.oed.com/view/Entry/30196?redirectedFrom=chai\#eid

6 http://www.oed.com/view/Entry/39596071?redirectedFrom=matcha\#eid

7 http://www.oed.com/view/Entry/58431?rskey=4GXjsi\&result=2\&isAdvanced=false\#eid

${ }^{8}$ Katakana is the Japanese phonetic script used for rendering imported and foreign words.

9 "Boomerang" words are those which have travelled from one country to another and back again.

\section{REFERENCES}

Bolton, K. (2003). Chinese Englishes: A sociolinguistic history. Cambridge: Cambridge University Press.

Chimuanya, L. \& Awonuga, C. (2015). A lexico-semantic study of culinary terms in Nigerian English: The question of intelligibility revisited. Covenant Journal of Language Studies. 3(1), 35-56.

Gerhardt, C., Frobenius, M. \& Ley, S. (Eds.). (2013). Culinary linguistics: The chef's special. Amsterdam: John Benjamins Publishing.

Jurafsky, D. (2014). The language of food: A linguist reads the menu. New York: W.W. Norton \& Company.

Kachru, B. B. (1994). Englishization and contact linguistics. World Englishes. 13(2), 135-154.

Kiaer, J. (2019). Translingual words: An East Asian lexical encounter with English. New York: Routledge.

Kiaer, J. \& Bordilovskaya, A. (2017). Hybrid English words in Korean and Japanese: A strange brew or an asset for global English?. Asian Englishes. 19(2), 169-187.

Loveday, L. J. (1996). Language contact in Japan: A socio-linguistic history. Oxford: Clarendon Press.

Platt, J. R., Weber, H. \& Ho, M. L. (1984). The New Englishes. London: Routledge.

Pride, J. B. (Ed.). (1982). New Englishes. Rowley: Newbury House Publishers.

Riley, K. C. \& Paugh, A. L. (2018). Food and language: Discourses and foodways across cultures. New York: Routledge.

Schneider, E. W. (2014). New reflections on the evolutionary dynamics of world Englishes. World Englishes. $33(1), 9-32$.

Stanlaw, J. (2004). Japanese English: Language and culture contact. Hong Kong: Hong Kong University Press. 